\title{
Search for new physics with the SHiP experiment at CERN
}

\author{
Oliver Lantwin* \\ Imperial College London, London, United Kingdom \\ E-mail: oliver.lantwinecern.ch
}

SHiP is a new general purpose fixed target experiment at the CERN SPS designed to complement LHC experiments in the search for new physics. In its initial phase, the $400 \mathrm{GeV}$ proton beam extracted from the SPS will be dumped on a heavy target with the aim of integrating $2 \times 10^{20}$ pot in 5 years. Shielded by an active muon shield, a dedicated detector, based on a long decay volume followed by a spectrometer and particle identification detectors, will allow probing a variety of models with light long-lived exotic particles with masses below $\mathscr{O}(10) \mathrm{GeV} / c^{2}$. The main focus will be the physics of the so-called Hidden Portals, i. e. search for Dark Photons, Light scalars and pseudo-scalars, and Heavy Neutral Leptons. The sensitivity to Heavy Neutral Leptons will allow for the first time to probe, in the mass range above the kaon mass, a coupling range for which Baryogenesis and active neutrino masses could also be explained. A dedicated emulsion-based detector will allow detection of light dark matter in an unexplored parameter range.

The European Physical Society Conference on High Energy Physics

5-12 July

Venice, Italy

${ }^{*}$ Speaker. 


\section{Introduction}

Particle physics is faced with a puzzle. Even though there is experimental evidence for new physics beyond the standard model (SM), with the exception of neutrino oscillations and indications from cosmology, so far all laboratory experiments have been incredibly consistent with the predictions of the standard model. This leaves us clueless as to where the SM breaks down and beyond standard model (BSM) physics takes over. And so far none of the predictions of popular BSM models have been confirmed.

There are two possibilities for why we did not see BSM physics yet: The LHC might not be powerful enough to explore the scale of new physics, which could be just around the corner, but could be out of reach of current and future technology as well. Alternatively, new physics could be too weakly coupled to the SM to be seen at general purpose experiments.

I focus on this second option here and will explain how Search for Hidden Particles (SHiP) [1] is designed to find these elusive particles, in particularly for super-weakly coupled new physics with $m_{\mathrm{NP}}<\mathscr{O}(10 \mathrm{GeV})$.

If there is super weakly coupled new physics, there generally is a portal that mediates between the standard model and one or more hidden particles, i.e. the hidden sector (HS):

$$
\mathscr{L}=\mathscr{L}_{\mathrm{SM}}+\mathscr{L}_{\text {portal }}+\mathscr{L}_{\mathrm{HS}}
$$

where $\mathscr{L}_{\mathrm{SM}}$ is the SM Lagrangian, $\mathscr{L}_{\mathrm{HS}}$ is the Lagrangian of a possibly richly structured hidden sector, and $\mathscr{L}_{\text {portal }}$ are the Lagrangian terms linking the two, i. e. those we could conceivably test for experimentally. If these interaction terms do exist, their mathematical form is constrained by the fact that they, by definition, also involve SM fields. The simplest possible interactions of this kind are tabulated in table 1 .

As an example to motivate the SHiP design, we will consider the heavy neutral lepton (HNL) of the neutrino minimal standard model $(v \mathrm{MSM})-\mathrm{a}$ fermion portal. For details on the many other models, the reader is referred to our physics proposal (PP) [2]. The $v$ MSM [3] is a model with a minimal number of additional particles that can solve all of the experimental shortcomings of the SM. In it three right handed neutrinos $N_{i}$ are added to complete the SM:

- A light $N_{1}$ with mass $\mathscr{O}(10 \mathrm{keV})$ is essentially decoupled from $N_{2,3}$, making it a dark matter candidate.

- Two heavy $N_{2,3}$ with masses $\mathscr{O}(1 \mathrm{GeV})$ mix with the active neutrinos, effectively coupling them weakly to the SM. They are the HNL. Through the mixing they set the active neutrino

\begin{tabular}{lr}
\hline Portal & Interaction term \\
\hline Scalar (e.g. dark scalar, dark Higgs) & $\left(H^{\dagger} H\right) \phi$ \\
Vector (e.g. dark photon) & $\varepsilon F_{\mu \nu} F_{\mu \nu}^{\prime}$ \\
Fermion (e. g. heavy neutral lepton (HNL)) & $H^{\dagger} \bar{N} L$ \\
Axion-like particle (ALP) & $a F^{\mu v} \tilde{F}^{\mu \nu}$ \\
\hline
\end{tabular}

Table 1: Possible portal interactions 
masses via the see-saw mechanism, and via leptogenesis they can also explain the baryon asymmetry of the universe (which is converted from an asymmetry of the leptons to baryons via sphaelerons). Importantly for experimental studies, they can be produced in heavy flavour decays, and can decay back to visible final states.

With this benchmark model in mind, we can turn to how SHiP is designed to look for this and other portal models.

\section{The SHiP experiment}

An overview of the SHiP facility is shown in figure 1. The SHiP experiment is designed to look for two types of signatures predicted by many new physics models:

1. Via decay to visible particles in hidden sector spectrometer,

2. Via scattering off electrons or nuclei in nuclear emulsion.

To ensure sensitivity to very weakly coupled new physics it is essential to maximise the intensity while minimising backgrounds. An intense proton beam from the super proton synchrotron (SPS) at $400 \mathrm{GeV}$ at the new beam dump facility (BDF) in the North Area will supply SHiP with $2 \times 10^{20}$ protons on target over 5 years. It impinges on a very dense target of $12 \times \lambda_{\text {int }}$, which ensures abundant heavy flavour production while reducing neutrino production from $\pi$ and $K$ decays. The yields of $D$-mesons and $\tau$-leptons over 5 years are expected to be in excess of $10^{18}$ and $10^{16}$ respectively. Additionally, there will be a high yield of photons from bremsstrahlung, QCD processes and meson decay, which allows the search for e. g. dark photons.

The number of protons extracted from the SPS will be similar to that provided for the CERN neutrinos to Gran Sasso (CNGS) project, but with slow instead of fast extraction of the beam. This will allow operation in parallel with the LHC and other beam-lines at the SPS.

With enough intensity to possibly produce new particles, the crucial challenge becomes rejecting backgrounds from SM processes, SHiP aims to be a zero background experiment for the visible decay signature in the hidden sector spectrometer.

The heavy target and the hadron absorber stop most SM particles, with the exception of muons and neutrinos. Since the decay volume is under a vacuum to prevent neutrino interactions within the fiducial volume, the muons become the key problem. As muons lose very little momentum in material, and the SHiP detectors need to be as close to the target as possible, active shielding is needed to deflect the muons away from the detectors. This active shield is comprised of a system of warm electro-magnets, which first separate the muon charge and then deflect them to either side. To achieve the goal of zero background it needs to reduce the flux of muons in the detector by at least six orders of magnitude, for the full kinematic range of muons produced, so up to $p \sim 350 \mathrm{GeV}$ and $p_{T} \sim 8 \mathrm{GeV}$, in as short a distance as possible. This makes the shield configuration critical to optimise, and a re-optimisation using detailed simulations and Bayesian Optimisation techniques is currently in progress. For more details on the current design, see Ref. [4]. For this optimisation an accurate knowledge of the muon spectrum is vital. To improve our confidence that the muon spectrum is modelled well in simulation a measurement of the muon spectrum for the SHiP target at the H4 test-beam at CERN's SPS is planned for 2018 [5]. 


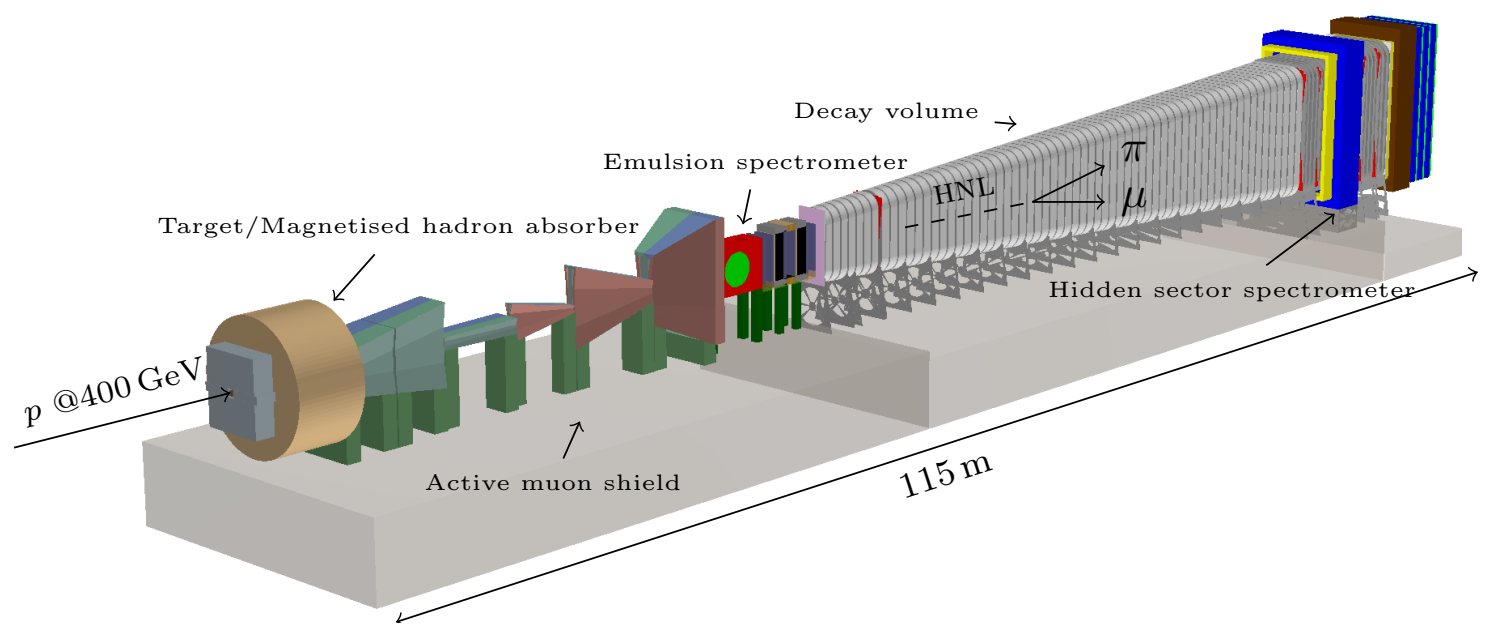

Figure 1: SHiP

To further reduce backgrounds from particles produced by muons passing through material, neutrino interactions in the surrounding structures and cosmic rays, the decay vessel is surrounded by background taggers, to detect any visible particles entering or exiting the vessel. A timing detector will further suppress combinatorial background, while tracking is used for vertexing and impact parameter measurement, further improving the rejection of fake signal candidates. Finally calorimeters and a muon detector allow particle identification, allowing study of specific final states. Some of the expected signal final states are tabulated in table 2. Taken together these subsystems are designed to redundantly reduce any possible backgrounds to negligible levels.

To search for hidden sector particles via the complementary signature of scattering, which is particularly important for e. g. light dark matter, and to study tau neutrinos, a detector based on nuclear emulsions is situated just downstream of the muon shield in front of the decay volume of the hidden sector spectrometer.

\section{SHiP Sensitivity}

This section will give a brief overview of the SHiP sensitivity to several classes of models compared to other current and future experiments. Please note, that these sensitivities are from

\begin{tabular}{lr}
\hline Particle & Final states \\
\hline HNL, neutralino & $\ell^{ \pm} \pi^{\mp}, \ell^{ \pm} K^{\mp}, \ell^{ \pm} \rho^{\mp}$ \\
Vector, scalar, axion portals; goldstino & $\ell^{ \pm} \ell^{\mp}$ \\
HNL, neutralino, axino & $\ell^{ \pm} \ell^{\mp} v_{\ell}$ \\
Axion portal, sgoldstino & $\gamma \gamma$ \\
Sgoldstino & $\pi^{0} \pi^{0}$ \\
\hline
\end{tabular}

Table 2: Visible final-states by hypothesised signal 
before the current round of re-optimisation, i. e. these sensitivity curves correspond to the technical proposal (TP) [1] configuration.

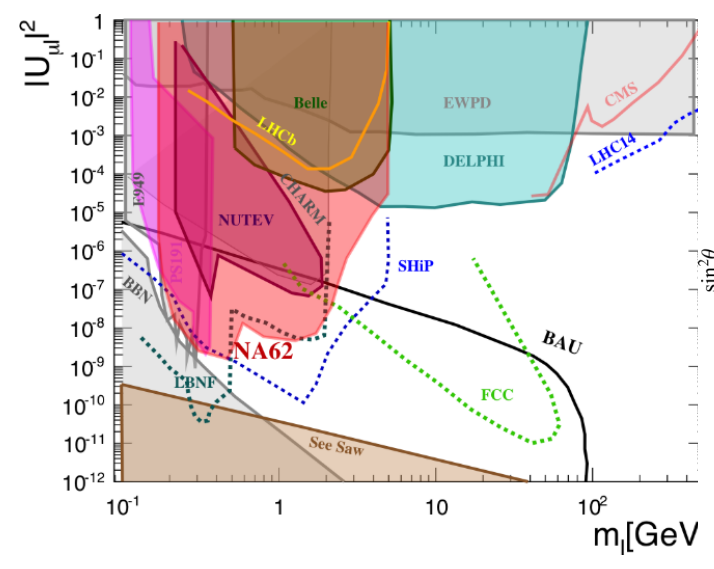

(a)

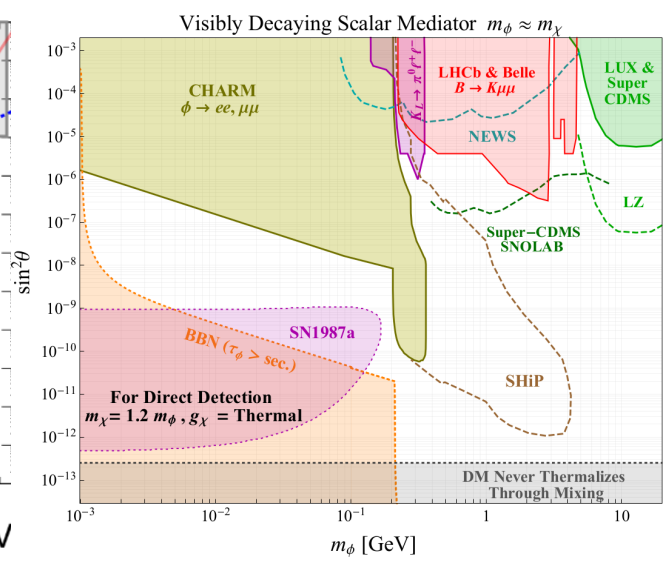

(b)

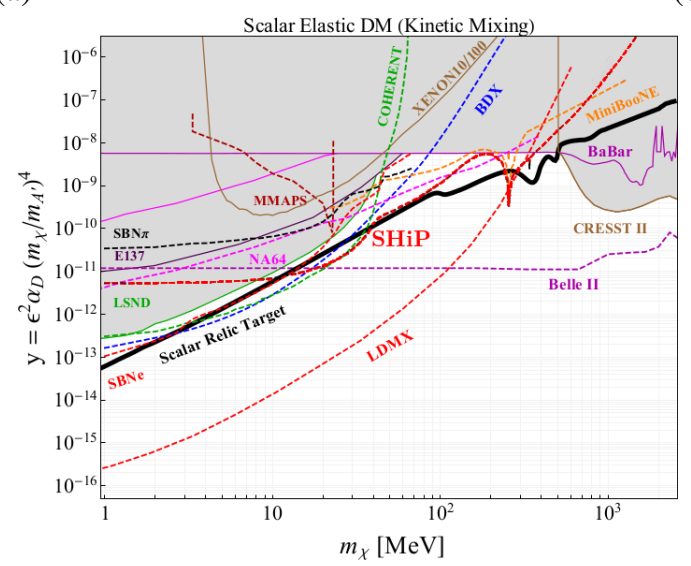

(c)

Figure 2: Sensitivity for different models at SHiP. (a) HNL sensitivity at SHiP for $v$ MSM with $U_{e}^{2}: U_{\mu}^{2}: U_{\tau}^{2}=1: 16: 3.8$ and a normal neutrino mass hierarchy. Source: Ref. [6]; (b) Dark scalar sensitivity at SHiP. Source: Ref. [7]; (c) Light dark matter sensitivity at SHiP for $\frac{m_{A^{\prime}}}{m_{\chi}}=3$. Source: Ref. [8]

\subsection{HNL}

For HNL the available parameter space is limited theoretically by observations of the baryon asymmetry of the universe (BAU), the big bang nucleosynthesis (BBN) and a model-independent limit for all see-saw models. The SHiP sensitivity for HNL in this space is shown in figure 2a.

The SHiP sensitivity is best up to about $3 \mathrm{GeV}$, which is above the charm kinematic limit, thanks to a significant contribution from $B$-meson decays. In this region it is unique and complementary to the region that could be probed at the future circular collider (FCC) in $e^{+} e^{-}$mode.

\subsection{Dark scalars}

The SHiP sensitivity for dark scalars is shown in figure 2b. Again, SHiP covers a unique part of the parameter space, complementary to other experiments. For short lifetimes $B$-factories and $\mathrm{LHCb}$ 
dominate. $B$-decays have a large contribution to the sensitivity achievable at SHiP. Note, that there is a gap at $c \tau \sim \mathscr{O}(\mathrm{m})$, where the lifetime is too short for SHiP and too long for the $B$-experiments, emphasising the importance for SHiP to be as close a possible to the target.

\subsection{Dark photons}

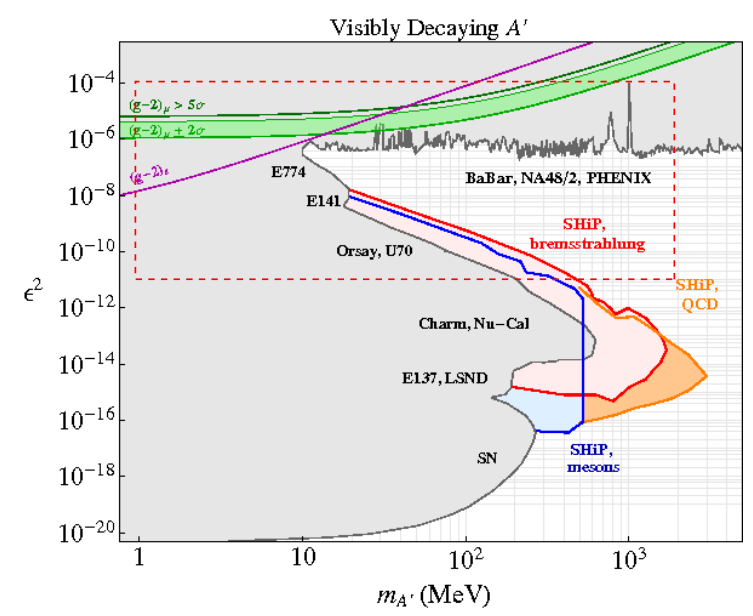

(a)

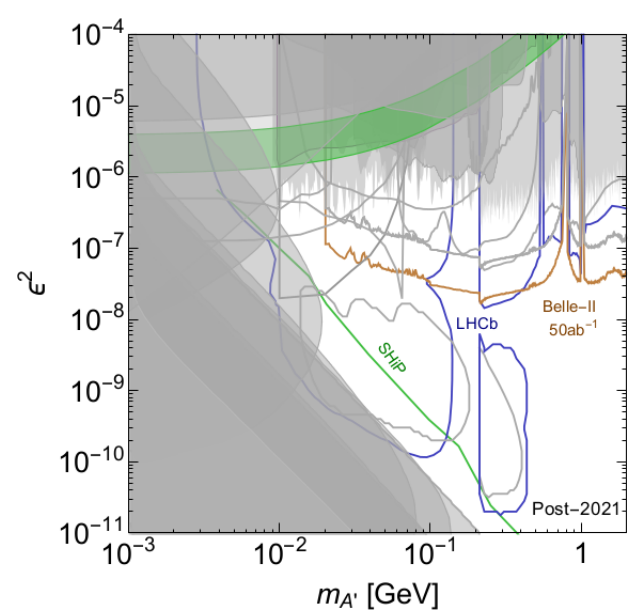

(b)

Figure 3: Dark photon sensitivity at SHiP. (a) Sensitivity to visible final states. Source: Ref. [2]; (b) Detail of highlighted region. Source: Ref. [9]

The SHiP sensitivity for dark photons is shown in figure 3. This estimate is based on a yield of $>10^{20} \gamma$ at SHiP over 5 years. The dark photons here decay to visible final states. The SHiP simulation includes production via $\mathrm{QCD}$, bremsstrahlung and meson decays, with the respective sensitivities shown separately in figure $3 \mathrm{a}$, but not yet via electromagnetic showers, which are currently being implemented.

The sensitivity of SHiP covers a broad region of the parameter space up to masses of a few $\mathrm{GeV}$ and couplings down to $10^{-16}$. The upper boundary of the SHiP sensitivity is determined by the detector's distance to the target, as dark photons would decay before reaching the decay volume. However, other existing and future experiments can explore this region at higher couplings which is shown in more detail in figure 3b, complementing the region explorable at SHiP.

\subsection{Light dark matter}

For dark matter lighter than WIMPs "direct detection" experiments quickly lose sensitivity, due to the small recoil energy, which requires a very low energy threshold of the detectors. The two common approaches to hunt for light dark matter are via missing mass/energy searches ${ }^{1}\left(\propto U^{2}\right)$ and via scattering/recoil $\left(\propto U^{4}\right)$, which are complementary.

At SHiP light dark matter is searched for indirectly via electron and nuclear recoil in nuclear emulsion. The main backgrounds here are electron recoils from $v_{e}$ scattering, but differences in

\footnotetext{
${ }^{1}$ Missing energy searches assume a dark photon mediator and are thus insensitive to light dark matter produced by other mediators.
} 
energy and angular spectra can be exploited to look for an excess consistent with light dark matter. The SHiP sensitivity for light dark matter is shown in figure 2c. Note that the sensitivity shown here is preliminary, as cascade production of light dark matter is not yet implemented. Furthermore, this sensitivity projection only considers electron recoil, while nuclear recoils are not yet included. Even though, SHiP already has the best sensitivity for scattering, complementing LDMX, which searches for light dark matter via missing energy at an electron beam.

\section{Conclusion}

There is plenty of unexplored parameter space in the dark sector new physics could hide in. SHiP is designed to be sensitive to many different final states for both decay and scattering, allowing it to probe a vast range of models.

Currently SHiP is being re-optimised to improve the physics performance further while respecting cost constraints. In this context the sensitivities and backgrounds are currently being re-evaluated and updated for new configurations. For the sensitivity updates in particular additional physics models, production and decay channels are being added in close collaboration with the theoretical community.

\section{References}

[1] SHiP Collaboration collaboration, M. Anelli et al., A Facility to Search for Hidden Particles (SHiP) at the CERN SPS, Tech. Rep. CERN-SPSC-2015-016. SPSC-P-350., CERN, Geneva, Apr, 2015.

[2] SHiP Collaboration collaboration, S. Alekhin et al., A facility to Search for Hidden Particles at the CERN SPS: the SHiP physics case, Tech. Rep. CERN-SPSC-2015-017. SPSC-P-350-ADD-1., CERN, Geneva, Apr, 2015.

[3] T. Asaka, S. Blanchet and M. Shaposhnikov, The nuMSM, dark matter and neutrino masses, Phys. Lett. B631 (2005) 151-156, [hep-ph / 0503065$].$

[4] SHIP collaboration, A. Akmete et al., The active muon shield in the SHiP experiment, JINST 12 (2017) P05011, [1703.03612].

[5] SHiP COllaboration collaboration, E. van Herwijnen, Muon-flux measurements for SHiP at H4, Tech. Rep. CERN-SPSC-2017-020. SPSC-EOI-016, CERN, Geneva, Jun, 2017.

[6] T. Spadaro, Perspectives from NA62, in Physics beyond colliders workshop @ CERN (7 Sep 2016), 2016, https://indico.cern.ch/event/523655/contributions/2246416/.

[7] G. Krnjaic, Probing Light Thermal Dark-Matter With a Higgs Portal Mediator, Phys. Rev. D94 (2016) 073009, [1512.04119].

[8] M. Battaglieri et al., US Cosmic Visions: New Ideas in Dark Matter 2017: Community Report, 1707.04591.

[9] J. Alexander et al., Dark Sectors 2016 Workshop: Community Report, 2016, 1608. 08632 , https://inspirehep.net/record/1484628/files/arXiv:1608.08632.pdf. 